\title{
Radiation therapy for intracranial tumours in cats with neurological signs
}

Körner, Maximilian ; Roos, Malgorzata ; Meier, Valeria Sabina ; Soukup, Alena ; Cancedda, Simona ; Parys, Magdalena M ; Turek, Michelle ; Rohrer Bley, Carla

\begin{abstract}
Objectives The aim of this study was to evaluate the outcome of cats with intracranial tumours presenting with neurological signs treated with radiation therapy. Methods This study comprised a retrospective multi-centre case series. Medical records of a total of 22 cats with intracranial spaceoccupying lesions, presenting with neurological signs and/or epileptic seizures and treated with external beam radiation therapy, were reviewed. In the treated cats, patient-, tumour- and treatment-related variables were investigated, including age, sex, tumour location, tumour volume, total radiation dose, equivalent dose in 2 Gy fractions (EQD2), corticosteroid dose, overall treatment time and institution for influence on local tumour control and survival. Results Based on advanced imaging characteristics, the 22 treated cats presented with meningioma $(n=11)$, pituitary tumour $(n=8)$, choroid plexus tumour ( $n$ $=2$ ) or glioma $(n=1)$. Allocated to the neuraxis, 11 lesions were extra-axial, three intra-axial and eight were located in the pituitary region. At diagnosis, 21 cats exhibited altered neurological status. One cat presented with epileptic seizures and another cat had both seizures and altered neurological status. The mean total physical dose of radiation was 41.63 Gy $( \pm 4.33)$, range $24-45$ Gy. In all but one cat (95.5\%), neurological signs improved after radiation therapy. The median progression-free survival was 510 days (95\% confidence interval [CI]: 51-969). The proportion free of progression at 1 year was $55.7 \%$ (95\% CI: 33-78). Fourteen cats died (only in five cases was death related to the intracranial tumour) and eight cats were still alive or lost to follow-up. The median overall survival time was 515 days (95\% CI: 66-964). None of the tested variables influenced outcome. Conclusion and relevance Radiation therapy seems to represent a viable treatment option in cats with intracranial tumours, relieving neurological signs and improving local tumour control. Radiation therapy may be considered for cats with tumours in complicated/inoperable localisations or for cases with a high peri- and postoperative risk
\end{abstract}

DOI: https://doi.org/10.1177/1098612x18801032

Posted at the Zurich Open Repository and Archive, University of Zurich

ZORA URL: https://doi.org/10.5167/uzh-158184

Journal Article

Accepted Version

Originally published at:

Körner, Maximilian; Roos, Malgorzata; Meier, Valeria Sabina; Soukup, Alena; Cancedda, Simona; Parys, Magdalena M; Turek, Michelle; Rohrer Bley, Carla (2019). Radiation therapy for intracranial tumours in cats with neurological signs. Journal of Feline Medicine and Surgery, 21(8):765-771.

DOI: https://doi.org/10.1177/1098612x18801032 


\section{Radiation therapy for Intracranial Tumors in Cats with Neurologic Signs}

Maximilian Körner ${ }^{1}$, Malgorzata Roos ${ }^{2}$, Valeria S. Meier ${ }^{1}$, Alena Soukup ${ }^{1}$, Simona Cancedda ${ }^{3}$, Magdalena M. Parys ${ }^{4}$, Michelle Turek ${ }^{5}$, Carla Rohrer Bley ${ }^{1}$

${ }^{1}$ Divison of Radiation Oncology, Vetsuisse Faculty, University of Zurich, CH-8057 Zurich,

Switzerland

${ }^{2}$ Department of Biostatistics, Epidemiology, Biostatistics and Prevention Institute, Faculty of Medicine, University of Zurich, CH-8001 Zurich, Switzerland

${ }^{3}$ Centro Oncologico Veterinario, 40037 Sasso Marconi (BO), Italy

${ }^{4}$ Royal (Dick) School of Veterinary Studies, Hospital for Small Animals, University of Edinburgh, Easter Bush Campus, EH25 9RE, Roslin, United Kingdom

${ }^{5}$ Department of Surgical Sciences, School of Veterinary Medicine, University of Wisconsin-Madison, Madison, USA

\section{Corresponding Author: med. vet. Maximilian Körner}

Division of Radiation Oncology

Vetsuisse Faculty, University of Zurich

Winterthurerstrasse $258 \mathrm{c}$

CH-8057 Zurich

Switzerland

Email: mkoerner@vetclinics.uzh.ch

Phone: +41446358324

Running head: Radiation for brain tumors in cats

Keywords: brain tumor, meningioma, glioma, feline, radiation therapy, intracranial, radiotherapy, pituitary 


\begin{abstract}
Objectives The objective of this study was to evaluate the outcome of cats with intracranial tumors presenting with neurological signs treated with radiation therapy.

Methods Retrospective multicenter case series. Medical records of a total of 22 cats with intracranial space-occupying lesions, presenting with neurologic signs and/or epileptic seizures, and treated with external beam radiation therapy, were reviewed. In the treated cats we investigated patient-, tumor- and treatment-related variables, including age, sex, tumor location, tumor volume, total radiation dose, $\mathrm{EQD}_{2}$, corticosteroid dose, overall treatment time and institution for influence on local tumor control and survival.
\end{abstract}

Results Based on advanced imaging characteristics, the 22 treated cats presented with meningioma $(n=11)$, pituitary tumor $(n=8)$, choroid plexus tumor $(n=2)$, or glioma $(n=1)$. Allocated to the neuraxis, 11 lesions were extra-axial, 3 intra-axial and 8 were located in the pituitary region. At diagnosis, 21 cats exhibited altered neurological status. One cat presented with epileptic seizures and another cat had both seizures and altered neurological status. The mean total physical dose of radiation was 41.63 Gy ( \pm 4.33), range $24-45 \mathrm{~Gy}$. In all but one cat (95.5\%), neurological signs improved after radiation therapy. The median progression free survival (PFS) was 510 days $(95 \% \mathrm{CI}$ : 51;969). The proportion free of progression at 1 year was $55.7 \%(95 \% \mathrm{CI}: 33 ; 78)$. Fourteen cats died (only in 5 cases death was related to the intracranial tumor) and 8 cats were still alive or lost to follow up. The median overall survival time (OS) was 515 days (95\%CI: 66;964). None of the tested variables influenced outcome.

Conclusion and relevance Radiation therapy seems to represent a viable treatment option in cats with intracranial tumors, relieving neurological signs and improving local tumor control. Radiation therapy may be considered for cats with tumors in complicated/inoperable localizations or for cases with a high peri- and postoperative risk. 


\section{Introduction}

Neurological signs in cats with primary intracranial tumor are common and include altered consciousness (36.8\%), circling (31.6\%), and epileptic seizures (31.6\%). ${ }^{1}$ Symptomatic therapy such as corticosteroids and/or antiepileptic medications provides only short-term relief. Prognosis in these conservatively managed patients is poor, with reported survival of 18 days. ${ }^{1-3}$ Complete or partial eradication of the tumor with surgery is desired in the management of intracranial disease and often results in improvement or resolution of neurologic signs.

The most frequently occurring brain tumor in cats is meningioma. ${ }^{1,4}$ Feline meningioma is often non-invasive (WHO grade 1) and located peripherally in the calvarium ${ }^{1,4}$, which often makes surgery the treatment of choice. Surgical excision results in favorable long-term outcomes with a median survival of 22.8-37 months. ${ }^{1,2,5}$ However, invasive tumors or tumors located at non-peripheral locations within the calvarium may be difficult to remove or may not be amenable to surgery. ${ }^{3}$ Treatment options for cats with inoperable intracranial tumors are not well described. In dogs with primary intracranial neoplasia of any type presenting with neurologic signs, radiation therapy is considered one of the treatments of choice. After radiation therapy, an improvement within 6 months or resolution of neurological signs associated with a long-term survival in the range of 2 years is observed in dogs with brain tumors. ${ }^{6-9}$ In cats, response to ionizing radiation and subsequent tumor control are not known. The few studies that describe response to radiation therapy in cats presented with neurological signs caused by an intracranial tumor are limited to pituitary tumors but indicate that neurologic amelioration can occur. ${ }^{10-13}$

In this retrospective multicenter cases series, we describe the progression free - and overall survival in cats with intracranial tumors presented with neurological signs after treatment with radiotherapy (RT). We investigated the influence of patient-, tumor- and treatment-related variables on local control and survival. 


\section{Materials and Methods}

\section{Patients}

The databases of the Division of Radiation Oncology of the Vetsuisse Faculty, University of Zurich (Switzerland), the Centro Oncologico Veterinario (Italy), the Royal (Dick) School of Veterinary Studies, Radiation Oncology Department, University of Edinburgh (UK) and the University of Wisconsin-Madison Veterinary Medical Teaching Hospital (USA) were searched for cats with primary, non-lymphoid intracranial space-occupying lesions with imaging diagnosis of a tumor, presented with neurologic signs that had been treated with radiation therapy alone between January 2002 and June 2017. Cats that had undergone surgery (complete excision or debulking) or received any type of systemic anticancer treatment such as chemotherapy were excluded from the study. Contributing veterinary radiation oncologists were asked to collect information from the medical record of each case including signalment, neurologic signs, diagnostic tests, tumor localization, and imaging-based diagnosis, medical management (i.e.

corticosteroids), radiation therapy protocol and survival time. The space-occupying lesions in the brain were diagnosed from board certified radiologist either by contrast-enhanced computed tomography (CT) or magnetic resonance imaging (MRI). Due to the lack of histological diagnosis, the lesions were allocated to one of three groups according to their imaging characteristics: A) extra-axial tumors (consistent with meningioma, schwannoma, and choroid plexus tumor), B) intra-axial tumors (consistent with glioma), and C) pituitary tumors (consistent with adenoma and adenocarcinoma of the pituitary gland). ${ }^{7}{ }^{14}$ A second grouping was made according to the location of the tumor relative to calvarial anatomy: 1) rostral cranial fossa (rostral to the optic chiasm), 2) middle cranial fossa (between the optic chiasm and the dorsum sellae and petrosal crests: pituitary/temporal region), and 3) caudal cranial fossa (between the dorsum sellae and petrosal crests and the foramen magnum: including brain stem and cerebellum). ${ }^{15}$ Lesions suspicious for lymphoma (based on diagnostic imaging criteria) were excluded. ${ }^{14}$ The patients neurologic signs at the time of diagnosis were grouped into 3 categories: A) patients with altered neurologic status (changes in mentation and behavior, gait and postural abnormalities, deficits of cranial nerves including central blindness and head tilt), B) patients with epileptic seizures only, C) patients with epileptic seizures and altered neurologic status. Other information recorded included medical treatment at the time of diagnosis, 
findings regarding tumor staging and if available, cerebrospinal fluid analysis.

\section{Treatment}

All cats were treated with external beam megavoltage radiation therapy. Radiation was delivered with 6 MV linear accelerators ${ }^{\mathrm{a}}$. Treatment planning was performed by a board-certified veterinary radiation oncologist in all cases, on the basis of 3D-CT datasets and using computerized radiation treatment planning systems ${ }^{\mathrm{b}}$. Treatment planning CT scans were performed with each patient in treatment position using a customized immobilization device according to each institution's standard practice. ${ }^{16}$ The gross tumor volume (GTV) was defined as the abnormal contrast-enhancing tissue on CT or MRI images. The clinical tumor volume (CTV) was added at the radiation oncologist's discretion and a planning target volume (PTV) was added to include a safety margin accounting for systematic and random uncertainties. Organs at risk (normal brain tissue and eyes) were contoured in all cats. Radiation treatment was planned isocentrically using photons and 3-dimensional conformal radiation therapy. Beam shaping devices such as lead blocks, multi-leaf collimators and wedges were used to improve homogeneity and to minimize dose to organs at risk. In general, the dose was prescribed according to the International Commission on Radiation Units and Measurements. ${ }^{17,18}$ Irradiation was delivered in various fractionated regimes.

\section{Follow-Up}

Contributing veterinary radiation oncologists gathered the post-radiotherapy follow-up information from the patients' records. If a follow-up diagnostic imaging study was available, tumor response was quantified according to the RECIST guidelines for dogs. ${ }^{19}$ The sum of diameters of all target lesions was calculated and reported as the baseline sum diameter. Complete remission (CR) was defined as the disappearance of all target lesions. Partial response (PR) was defined as a reduction of at least $30 \%$ in the sum of diameters of target lesions from baseline. Progressive disease (PD) was defined as an increase in the sum of diameters of target lesions by at least $20 \%$ over baseline, or the appearance of new lesions. Tumors that neither showed sufficient shrinkage to qualify for PR nor sufficient increase to qualify for PD were considered to be of stable disease (SD). For cats that were not re-evaluated at one of the participating centers, the referring veterinarians and owners were queried by telephone regarding performance status, 
neurologic signs compared to those at the time of diagnosis, disease progression and date of death by one of the authors (MKO, MPA or SCA).

\section{Statistical Analysis}

Statistical evaluation was performed under the supervision of a biostatistician (MR) and computed with a commercial statistical software package (IBM ${ }^{\circledR}$ SPSS $^{\circledR}$ Statistics, Version 24). Data was used as submitted in a excel table by the contributing veterinary radiation oncologists. Description of quantitative data characteristics, other than progression-free survival (PFS) and overall survival (OS), is given by mean ( \pm SD), unless otherwise specified. Description of qualitative characteristics is provided in absolute and relative frequencies. PFS was defined as the interval between the first day of radiation therapy to measurable progression of disease, worsening of neurologic signs compared to the neurologic status before RT or death. OS was defined as the interval between the first fraction of radiation therapy and death. Cats that were free of progression of measurable tumor and of progression of neurologic signs and that were alive at the time of data collection were censored for PFS analysis. For OS, all deaths were considered events and cats that were still alive at the time of data collection or lost to follow-up were censored at the last date of contact. Kaplan-Meier survival analysis was used and followed by Logrank test. The univariate Cox-regression analysis was used to determine whether the following factors were associated with PFS or OS: age, sex, meningioma/ non-meningioma, meningioma/ pituitary tumor, tumor volume (GTV, GTV/brain ratio), treatment volume (PTV, PTV/brain ratio), tumor location, treatment institution, dose of radiation (total dose, $\mathrm{EQD}_{2}$ ), dose of corticosteroids at first RT fraction, overall treatment time (counted from the first to the last fraction of RT). The equivalent dose in 2 Gy fractions $\left(\mathrm{EQD}_{2}\right)$ was used to compare the different radiation protocols. Survival estimates at a certain time point are presented as percentage with the corresponding $95 \%$ confidence intervals $(95 \% \mathrm{CI})$. Median survival time estimates are complemented with $95 \% \mathrm{CI}$. The paired Wilcoxon test evaluated the change in corticosteroid dose from the first to the last RT fraction. Results of statistical analyses with p-value $<0.05$ were interpreted as statistically significant. 


\section{Results}

\section{Patient and tumor characteristics}

Twenty-two cats met the inclusion criteria for this study. Nine of the cats were female (9 spayed) and 13 were male (12 neutered). The presented breeds included domestic short-hair $(n=18)$, Persian $(n=3)$ and Selkirk Rex $(n=1)$. The mean age was 12.0 years $( \pm 2.3)$ and ranged from $6.5-16.4$ years. The mean body weight was $4.5 \mathrm{~kg}( \pm 1.4)$ and ranged from $2.3-8.3 \mathrm{~kg}$. Fifteen animals were treated at the Division of Radiation Oncology of the Vetsuisse Faculty, University of Zurich, Switzerland, 6 animals at the Centro Oncologico Veterinario, Sasso Marconi, Bologna, Italy, and 1 animal was treated at the Royal (Dick) School of Veterinary Studies at the Radiation Oncology of the University of Edinburgh, United Kingdom. There were no cats that met the inclusion criteria from the University of Wisconsin-Madison Veterinary Medical Teaching Hospital, USA.

Based on imaging characteristics, tumors were diagnosed as meningioma $(n=11)$, pituitary tumor $(n=8)$, choroid plexus tumor $(n=2)$, or glioma $(n=1)$. Eleven tumors were considered extra-axial, 3 intraaxial and 8 were located in the pituitary region. Tumors were distributed in the cranial fossa $(n=9)$, middle fossa $(n=8)$ or caudal fossa $(n=5)$. The pretreatment tumor volumes (GTV, absolute tumor size) had a mean of $1.78 \mathrm{~cm}^{3}( \pm 1.67)$ with a range from 0.15 to $7.70 \mathrm{~cm}^{3}$. Mean GTV/brain ratio [relative tumor size, $(\mathrm{n}=16)]$ was $8.32 \%( \pm 8.55)$ and the PTV/brain ratio could be derived for 16 cats with a mean of $33.18 \%$ $( \pm 34.00)$. The majority of cats presented with altered neurologic status and without epileptic seizures $(n=20)$. One cat presented with epileptic seizures only (Table 1, case no. 22) and one cat with both altered neurological status and epileptic seizures (Table 1, case no. 21).

\section{Treatment}

Twenty-one patients were treated with various definitive-intent daily-fractionated protocols (Table 1). One patient (Table 1, case no. 20) was treated with a highly conformal definitive-intent hypofractionated radiation protocol (3x8Gy) delivered every other day and received less than 40.00 Gy of total dose. Total doses ranged from 24.00 to $45.00 \mathrm{~Gy}$, with a mean total dose $41.63 \mathrm{~Gy}( \pm 4.33)$. Fraction sizes ranged from 2.25 to $8.00 \mathrm{~Gy}$, with a mean of $3.32 \mathrm{~Gy}( \pm 1.29)$. Twenty-one cats were treated using a daily (Monday-Friday) treatment schedule. Only case no. 20 (Table 1) was treated on a Monday-Wednesday- 
Friday schedule. All cats completed the full course of the planned radiation therapy. The respective $\mathrm{EQD}_{2}$ were 36.00 to $49.70 \mathrm{~Gy}_{2}$, with a mean of $45.79 \mathrm{~Gy}_{2}( \pm 2.74)$. Mean overall treatment time was 19.6 days (5 - 29 days). Pretreatment corticosteroid treatment with prednisolone was given to 20/22 cats with a mean dose of $0.66 \mathrm{mg} / \mathrm{kg}( \pm 0.41)$ once daily orally and tapered to mean doses of $0.58 \mathrm{mg} / \mathrm{kg}( \pm 0.43)$ once daily orally post RT ( $\mathrm{p}=0.076$ ). Three cats received antiepileptic treatment consisting of either phenobarbital $(n=2)$ or levetiracetam $(n=1)$ pre-, during and post RT. Two of these cats had epileptic seizures at initial presentation and one received phenobarbital prophylactically and was tapered off the medication after RT, as no epileptic seizures were observed.

\section{Outcome and prognostic factors}

Median follow-up time for censored cases ( $\mathrm{n}=8$, still alive or lost to follow-up) was 575 days (95\%CI: 464-686). Fourteen of the 22 cats died during the study period. Death was attributed to the brain tumor or possible treatment related toxicity in 5 of the 14 cats $(35.7 \%)$. In the $64.3 \%$ of animals that died due to other causes, death was related to other neoplastic disease $[n=4$, histiocytic sarcoma in the liver (Table 1 , case no. 4), lymphosarcoma in the bladder (Table 1, case no. 5), oral sarcoma (Table 1, case no. 10), intestinal lymphoma (Table 1, case no. 13)], tumor-unrelated medical problems (not accompanied with neurologic symptoms) like hyperthyroidism (Table 1, case no. 11), cardiac failure with dilated atria, arrhythmia and mild pericardial effusion (Table 1, case no. 14) or pyothorax (Table 1, case no. 19). In two cases (Table 1, case no. 3 and 17) the cats died at home with general weakness. Tumor recurrence could not be ruled out, because no necropsy was performed so the real cause for death remains unknown in these two cases.

Based on medical records and owner communication, clinical and neurologic status improved in 21/22 $(95.5 \%)$ cats, compared to the pre-treatment complaints. One of the 22 cats was judged to have stable clinical signs (Table 1, case no. 18). In the patient, in which epileptic seizures was the only presenting complaint, it was difficult to determine whether seizure control was attributable to reduction of tumor/edema or to anti-epileptic medication. 
A total of 10 post-radiotherapy diagnostic imaging examinations were performed in 6 patients. CT was performed 4 times ( $\mathrm{n}=3$ cats), and MRI was performed in 6 times ( $\mathrm{n}=3$ cats). On MRI one cat with a meningioma showed partial remission at 6 months post RT (Table 1, case no. 1), a second cat with a meningioma showed stable disease at 3,6 and 12 months post RT (Table 1, case no. 6). Another cat with a pituitary gland tumor showed stable disease on MRI at 6 and 12 months post RT (Table 1, case no. 12). In the CT of one cat 6 months post RT the tumor was in partial remission (the 12 months re-check CT of the same cat was made at another clinic and tumor volume was not measured) (Table 1, case no.15). Tumors of two cats were measured 24 months post RT, one cat still showed stable disease (Table 1, case no. 17) and the other cat was scanned upon re-occurrence of neurologic signs and had a disease progression on CT (Table 1, case no. 3). Overall, 7 cats $(31.8 \%)$ were considered to have a disease progression either on increased volume based on imaging $(n=1)$ or on progression of neurological signs $(n=6)$.

The median progression free survival (PFS) time for all 22 cases was 510 days (95\%CI: 51;969). The proportion free of progression at 1 year was 55.7\% (95\%CI: 33;78) (Figure 1). Of the 9 cats that died within the first year after RT, 7 (77.8\%) died of causes un-related to the brain tumor (Table 1, case no. 4, $5,10,11,13,14$ and 19$)$ and $2(22.2 \%)$ died because of the brain tumor or treatment related toxicity (Table 1, case no. 18 and 21). No difference was seen in PFS between the patients treated at the different institutions $(\mathrm{p}=0.634)$. At the time of tumor progression, two cats were re-irradiated resulting in an additional progression-free interval of 223 and 281 days (Table 1, case no. 3 and 17). Both cases were censored from analysis of OS at the start of the second course of radiation therapy. Neither the patient related (age, sex), tumor related (meningioma/ non-meningioma, meningioma/ pituitary tumor, tumor size, tumor location) or the treatment related parameters (dose of radiation [total radiation dose or $\mathrm{EQD}_{2}$ ], corticosteroid dose at first RT, overall treatment time, treatment institution) were associated with PFS.

Median overall survival time (OS) was 515 days (95\%CI: 66;964). The proportion alive at 1 year was $56 \%(95 \% \mathrm{CI}: 33 ; 79)$ and at 2 years $37 \%(95 \% \mathrm{CI}$ : 15;60) (Figure 2). No difference was seen in OS between the patients treated at the different institutions $(\mathrm{p}=0.695)$. None of the tested variables had a statistically detectable influence on OS. 


\section{Discussion}

Intracranial tumors causing neurological signs in cats are often of extra-axial and non-infiltrative nature and can be successfully treated with surgery. ${ }^{1-5}$ Meningiomas with and without neurological signs are the most common intracranial tumors in cats and postoperative median survival times were found to range between 22.8 to 37 months. ${ }^{1,2,5}$ For feline patients with tumors in less accessible locations such as tentorially located meningiomas, survival time was found to decrease to 19 months postoperatively. ${ }^{3}$ The risk of fatal intra- or postoperative complications ranged between $6-19 \% \%^{2,3,5}$ and depending on primary tumor location, the postoperative recurrence rate was $12-21 \%{ }^{1,2,5}$ and $100 \%^{3}$. In these retrospective studies should be a selection bias considered of only including surgically amenable tumors.

For cats with inoperable tumors however, literature findings are scarce and limited to cases of pituitary neoplasia and a case report of a cat with oligodendroglioma treated with radiation therapy. ${ }^{10-13,20}$ Surprisingly, amelioration of neurologic symptoms was rapid and often complete in most cats and survival times increased from a median of 1.1-1.7 months if treated with corticosteroids only ${ }^{1}$ to a range of 5.520.5 months after radiation. ${ }^{10-13}$ These results are difficult to compare to our findings, as in the cats treated for pituitary tumors only $38.9 \%$ had neurologic symptoms and most of them were treated because of pituitary-dependent endocrine disease. However, as it is known from dogs with pituitary tumors treated with radiation therapy, radiation therapy prolonged survival also in non-neurologic patients by delaying the onset of neurologic signs. ${ }^{21}$ In dogs with an intracranial primary tumor and neurologic signs, fractionated radiation therapy is often considered the treatment of choice with durable tumor control in the range of 19.2-25.2 months. ${ }^{6-9}$

In this study, the outcomes of cats with intracranial tumors presented with neurological signs were retrospectively compiled. Cases were sought from 4 institutions, and only 3 institutions could contribute patients. This fact indicates the scarcity of brain tumors in cats. After radiation therapy, the neurological status improved in all but one cat (95.5\%). Median overall survival time was 17.2 months, with $56 \%$ of cats alive at one year after therapy. Surprisingly, only seven patients (32\%) were considered to have disease progression during the time of follow-up. Most patients (9/14) died of other neoplastic diseases or unrelated medical problems. Upon recurrence of disease, two cats in this cohort were re-irradiated and 
attained an additional progression-free interval of 7.4 and 9.2 months (Table 1, case no. 3 and 17). None of the investigated factors such as age, sex, treatment institution, tumor location (extra-axial, intra-axial, pituitary), tumor type, tumor size, regions of brain affected, total dose, $\mathrm{EQD}_{2}$, corticosteroid dose at first RT, nor overall treatment time were found to correlate with progression-free or overall survival time.

We acknowledge limitations of this study that should direct future research approaches: A) The cats were treated at different institutions, with various non-standardized radiation protocols in terms of fraction size, total dose, overall treatment times, target volumes and treatment planning practices, equipment and patient position verification. In order to not skew the data into a direction, we decided to keep patients treated with all fraction schedules in the investigated group. B) Standardized neurological evaluation, severity of neurological signs, and routine follow-up diagnostic imaging were not available, which should constitute an important focus for further evaluation of tumor response to radiation therapy. C) Improvement of the clinical signs could - at least in part - also be attributed to medical supportive treatment such as corticosteroids. D) The patient population consisted of a heterogeneous group, with varying unconfirmed tumor types. Presumptive tumor diagnoses were made according to advanced diagnostic imaging techniques. ${ }^{14}$ Most of the tumors were meningiomas (50\%) or pituitary adenomas (36\%), which are commonly non-infiltrative and hence less aggressive. Furthermore, due to the limited number of patients, statistical analysis for each tumor type was not performed. E) The patients were referred for radiation therapy and the possibility of resectability was not uniformly judged. F) Due to the retrospective nature of the study and the lack of follow up imaging it was not in every case possible to differentiate tumor progression from radiation toxicity as the cause of recurring neurologic signs.

In conclusion, findings of this study suggest radiation therapy as viable treatment option for cats with intracranial tumors presented with neurological signs. Case selection should focus on inoperable disease or patients with an estimated high peri- and postoperative risk.

\section{Funding}

No funding was provided 


\section{Conflict of interest}

The authors declare no conflict of interest related to this study.

Figure Legends:

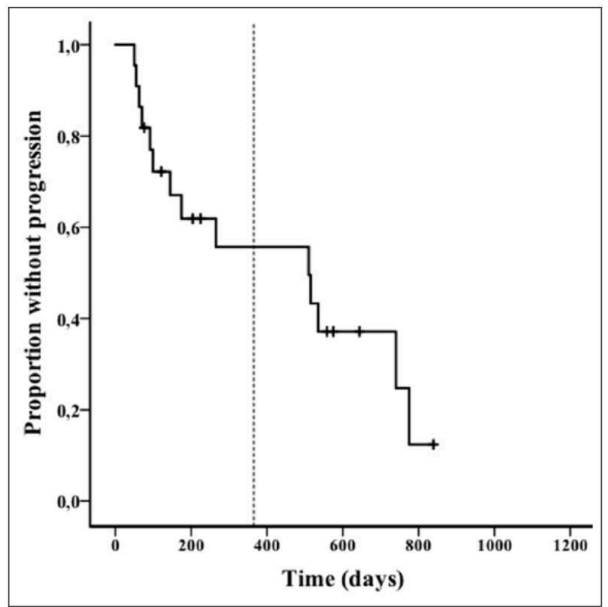

Figure 1: Median progression free survival (PFS). The dotted line marks 1-year. Tick marks: cats that were free of progression of measurable tumor and of progression of neurologic signs and that were alive at the time of data collection were censored for PFS analysis. Of the 9 cats that died within the first year after RT, 7 (77.8\%) died of causes un-related to the brain tumor and $2(22.2 \%)$ died because of the brain tumor or treatment related toxicity.

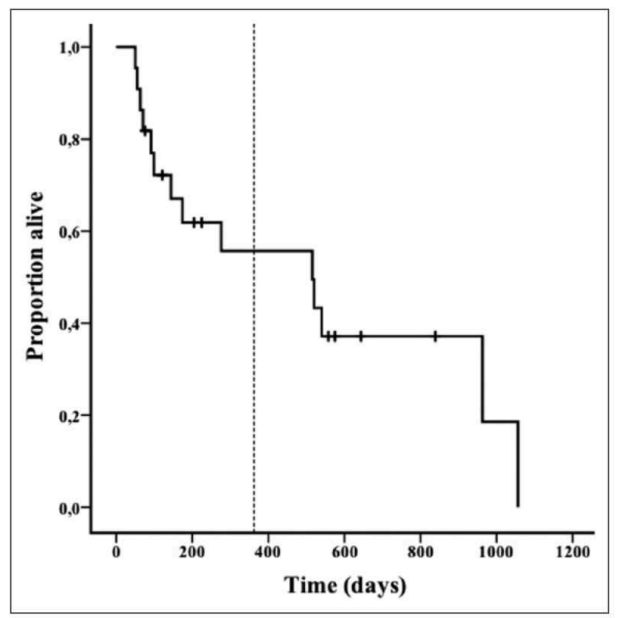

Figure 2: Median overall survival (OS): The dotted line marks 1-year. Tick marks: cats that were still alive at the time of data collection or lost to follow-up were censored at the last date of contact. 
Table 1: Individual patient data regarding tumor type and size, radiation protocols, progression free survival and overall survival

\begin{tabular}{|c|c|c|c|c|c|c|c|c|c|c|c|}
\hline No & $\begin{array}{c}\begin{array}{c}\text { Age } \\
\text { [years] }\end{array} \\
\text {. }\end{array}$ & Sex & $\begin{array}{l}\text { Tumor } \\
\text { type }\end{array}$ & $\begin{array}{l}\text { GTV } \\
{\left[\mathbf{c m}^{3}\right]}\end{array}$ & $\begin{array}{l}\text { No. of } \\
\text { fractions }\end{array}$ & $\begin{array}{l}\text { Treatment } \\
\text { schedule }\end{array}$ & $\begin{array}{c}\text { Dose/fraction } \\
\text { [Gy] }\end{array}$ & $\begin{array}{l}\text { Total dose } \\
\text { [Gy] }\end{array}$ & $\begin{array}{l}\text { EQD2 } \\
{\left[\mathrm{Gy}_{2}\right]}\end{array}$ & $\begin{array}{c}\text { PFS } \\
\text { [days] }\end{array}$ & $\begin{array}{c}\text { os } \\
\text { [days] }\end{array}$ \\
\hline 1 & 8 & $\mathrm{mn}$ & Meningioma & 4.19 & 10 & daily & 4 & 40 & 46.7 & $204 \dagger$ & $204 \uparrow$ \\
\hline 2 & 13 & $\mathrm{mn}$ & Meningioma & 0.51 & 10 & daily & 4 & 40 & 46.7 & $121 \dagger$ & $121 \dagger$ \\
\hline 3 & 11 & fs & Meningioma & 0.86 & 14 & daily & 3 & 42 & 45.5 & 740 & $963^{*}$ \\
\hline 4 & 11 & fs & Meningioma & 1.60 & 18 & daily & 2.5 & 45 & 46.9 & 50 & 50 \\
\hline 5 & 14 & fs & Meningioma & 0.15 & 14 & daily & 3 & 42 & 45.5 & 63 & 63 \\
\hline 6 & 12 & $\mathrm{mn}$ & Meningioma & 0.61 & 19 & daily & 2.25 & 42.75 & 43.6 & $225+$ & $225+$ \\
\hline 7 & 10 & $\mathrm{mn}$ & Meningioma & 3.49 & 20 & daily & 2.25 & 45 & 45.9 & 535 & $540^{\circ}$ \\
\hline 8 & 10 & $\mathrm{mn}$ & Meningioma & 2.35 & 20 & daily & 2.25 & 45 & 45.9 & 515 & 520 \\
\hline 9 & 11 & fs & Meningioma & 7.70 & 20 & daily & 2.25 & 45 & 45.9 & 510 & $515^{\circ}$ \\
\hline 10 & 14 & fs & Meningioma & 1.10 & 14 & daily & 2.9 & 40.6 & 43.6 & 91 & 91 \\
\hline 11 & 14 & $\mathrm{mn}$ & Meningioma & 2.35 & 20 & daily & 2.25 & 45 & 45.9 & 174 & 174 \\
\hline 12 & 13 & $\mathrm{mn}$ & $\begin{array}{c}\text { Pituitary } \\
\text { Gland Tumor }\end{array}$ & 2.55 & 10 & daily & 4 & 40 & 46.7 & $839 \dagger$ & $839 \dagger$ \\
\hline 13 & 16 & $\mathrm{mn}$ & $\begin{array}{c}\text { Pituitary } \\
\text { Gland Tumor }\end{array}$ & 0.72 & 10 & daily & 4.2 & 42 & 49.7 & 55 & 55 \\
\hline
\end{tabular}

\begin{tabular}{|c|c|c|c|c|c|c|c|c|c|c|c|}
\hline 14 & 13 & $\mathrm{mn}$ & $\begin{array}{c}\text { Pituitary } \\
\text { Gland Tumor }\end{array}$ & 1.17 & 14 & daily & 3 & 42 & 45.5 & 70 & 70 \\
\hline 15 & 12 & fs & $\begin{array}{c}\text { Pituitary } \\
\text { Gland Tumor }\end{array}$ & 0.82 & 10 & daily & 4.2 & 42 & 49.7 & $644 \dagger$ & $644 \dagger$ \\
\hline 16 & 7 & $\mathrm{~m}$ & $\begin{array}{l}\text { Pituitary } \\
\text { Gland Tumor }\end{array}$ & 1.42 & 17 & daily & 2.5 & 42.5 & 44.3 & $76 \dagger$ & $76^{\dagger}$ \\
\hline 17 & 12 & $\mathrm{mn}$ & $\begin{array}{c}\text { Pituitary } \\
\text { Gland Tumor }\end{array}$ & 1.20 & 14 & daily & 3 & 42 & 45.5 & 775 & $1056^{*}$ \\
\hline 18 & 12 & $\mathrm{mn}$ & $\begin{array}{c}\text { Pituitary } \\
\text { Gland Tumor }\end{array}$ & 2.10 & 14 & daily & 3 & 42 & 45.5 & 144 & 144 \\
\hline 19 & 11 & $\mathrm{fs}$ & $\begin{array}{c}\text { Pituitary } \\
\text { Gland Tumor }\end{array}$ & 1.44 & 20 & daily & 2.25 & 45 & 45.9 & 99 & 99 \\
\hline 20 & 12 & $\mathrm{mn}$ & $\begin{array}{l}\text { Choroid } \\
\text { Plexus Tumor }\end{array}$ & 0.17 & 3 & MWF & 8 & 24 & 36.0 & $558 \dagger$ & $558 \dagger$ \\
\hline 21 & 15 & fs & $\begin{array}{l}\text { Choroid } \\
\text { Plexus Tumor }\end{array}$ & 1.60 & 10 & daily & 4 & 40 & 46.7 & 265 & $276^{\circ}$ \\
\hline 22 & 14 & fs & Glioma & 1.01 & 10 & daily & 4.2 & 42 & 49.7 & $575 \dagger$ & $575 \dagger$ \\
\hline
\end{tabular}

$m$ : male; $m n$ : male neutered; $f s$ : female spayed; GTV: gross tumor volume; Gy: gray; $E Q D_{2}:$ equivalent dose delivered in 2 Gy fractions; $P F S$ : progression free survival; OS: overall survival; $\uparrow$ : cats free of progression and alive at the time of data evaluation or lost to follow-up were censored; *: cats were re-irradiated at the time of progression and censored from analysis of $\mathrm{OS}$ at the start of the second course of radiation therapy; $*$ : tumor type is based on advanced imaging characteristics, MWF: Monday-Wednesday-Friday, $\bullet$ : died of tumor or treatment-related causes 


\section{References:}

1. Troxel MT, Vite CH, Van Winkle TJ, et al. Feline intracranial neoplasia: retrospective review of 160 cases (1985-2001). J Vet Intern Med. 2003; 17: 850-9.

2. Cameron S, Rishniw M, Miller AD, Sturges B and Dewey CW. Characteristics and Survival of 121 Cats Undergoing Excision of Intracranial Meningiomas (1994-2011). Vet Surg. 2015; 44: 772-6.

3. Forterre F, Fritsch G, Kaiser S, Matiasek K and Brunnberg L. Surgical approach for tentorial meningiomas in cats: a review of six cases. J Feline Med Surg. 2006; 8: 227-33.

4. Tomek A, Cizinauskas S, Doherr M, Gandini G and Jaggy A. Intracranial neoplasia in 61 cats: localisation, tumour types and seizure patterns. J Feline Med Surg. 2006; 8: 243-53.

5. Gordon LE, Thacher C, Matthiesen DT and Joseph RJ. Results of craniotomy for the treatment of cerebral meningioma in 42 cats. Vet Surg. 1994; 23: 94-100.

6. Keyerleber MA, McEntee MC, Farrelly J, Thompson MS, Scrivani PV and Dewey CW. Threedimensional conformal radiation therapy alone or in combination with surgery for treatment of canine intracranial meningiomas. Vet Comp Oncol. 2015; 13: 385-97.

7. Rohrer Bley C, Sumova A, Roos M and Kaser-Hotz B. Irradiation of brain tumors in dogs with neurologic disease. J Vet Intern Med. 2005; 19: 849-54.

8. Treggiari E, Maddox TW, Goncalves R, Benoit J, Buchholz J and Blackwood L. Retrospective Comparison of Three-Dimensional Conformal Radiation Therapy Vs. Prednisolone Alone in 30 Cases of Canine Infratentorial Brain Tumors. Vet Radiol Ultrasound. 2017; 58: 106-16.

9. Schwarz P, Meier V, Soukup A, et al. Comparative evaluation of a novel, moderately hypofractionated radiation protocol in 56 dogs with symptomatic intracranial neoplasia. J Vet Intern Med. 2018; accepted.

10. Sellon RK, Fidel J, Houston R and Gavin PR. Linear-accelerator-based modified radiosurgical treatment of pituitary tumors in cats: 11 cases (1997-2008). J Vet Intern Med. 2009; 23: 1038-44.

11. Mayer MN, Greco DS and LaRue SM. Outcomes of pituitary tumor irradiation in cats. $J$ Vet Intern Med. 2006; 20: 1151-4. 
12. Kaser-Hotz B, Rohrer CR, Stankeova S, Wergin M, Fidel J and Reusch C. Radiotherapy of pituitary tumours in five cats. J Small Anim Pract. 2002; 43: 303-7.

13. Brearley MJ, Polton GA, Littler RM and Niessen SJ. Coarse fractionated radiation therapy for pituitary tumours in cats: a retrospective study of 12 cases. Vet Comp Oncol. 2006; 4: 209-17.

14. Troxel MT, Vite $\mathrm{CH}$, Massicotte $\mathrm{C}$, et al. Magnetic resonance imaging features of feline intracranial neoplasia: retrospective analysis of 46 cats. J Vet Intern Med. 2004; 18: 176-89.

15. Koblik PD, LeCouteur RA, Higgins RJ, et al. Modification and application of a Pelorus Mark III stereotactic system for CT-guided brain biopsy in 50 dogs. Vet Radiol Ultrasound. 1999; 40: 424-33.

16. Rohrer Bley C, Blattmann H, Malgorzata R, Sumova A and Kaser-Hotz B. Assessment of a Radiotherapy Patient Immobilization Device using Single Plane Port Radiographs and a Remote Computed Tomography Scanner Vet Radiol Ultrasound. 2003; 44: 470-5.

17. International Commission on Radiation Units and Measurements. Prescribing, Recording, and Reporting Photon Beam Therapy (Report 50). Bethesda, MD1993.

18. International Commission on Radiation Units and Measurements. Prescribing, Recording, and Reporting Photon Beam Therapy (Report 62, Supplement to ICRU Report 50). Bethesda, MD1999. 19. Nguyen SM, Thamm DH, Vail DM and London CA. Response evaluation criteria for solid tumours in dogs (v1.0): a Veterinary Cooperative Oncology Group (VCOG) consensus document. Veterinary and comparative oncology. 2015; 13: 176-83.

20. Tamura M, Hasegawa D, Uchida K, et al. Feline anaplastic oligodendroglioma: long-term remission through radiation therapy and chemotherapy. J Feline Med Surg. 2013; 15: 1137-40.

21. Kent MS, Bommarito D, Feldman E and Theon AP. Survival, neurologic response, and prognostic factors in dogs with pituitary masses treated with radiation therapy and untreated dogs. $J$ Vet Intern Med. 2007; 21: 1027-33.

\footnotetext{
a Dynaray LA20; ABB/Varian, Clinac DMX or Clinac iX, Varian Medical Systems, Palo Alto, CA, USA (Switzerland, Italy), Clinac 2100 C/D, Varian Medical Systems, Palo Alto, CA, USA (United Kingdom)
} 
${ }^{\mathrm{b}}$ CADPLAN $^{\mathrm{TM}}$ (precursor versions of Eclipse ${ }^{\mathrm{TM}}$ ), Eclipse ${ }^{\mathrm{TM}}$ External Beam Planning system version 8.6, 10.0 and 11.0 (Varian Oncology Systems, Palo Alto, CA, USA) 